\title{
INFLUÊNCIA DA FLEXIBILIDADE NO DESEMPENHO EM SALTOS VERTICAIS
}

\section{Influence of flexibility in the performance in vertical jumps}

\author{
Bruno Pena Couto ${ }^{1,2}$, Hosanna Rodrigues Silva ${ }^{1,2}$, Caroline Regiane Cunha ${ }^{3}$, Flávia Mansur de Aguiar Cotting ${ }^{3}$, Natália Vitor \\ de Alcântara ${ }^{3}$ \\ ${ }^{1}$ Laboratório de Bioengenharia, Escola de Engenharia - Universidade Federal de Minas Gerais \\ Belo Horizonte - MG - Brasil. \\ ${ }^{2}$ Laboratório de Avaliação da Carga, Escola de Educação Física, Fisioterapia e Terapia Ocupacional - Universidade Federal de \\ Minas Gerais - Belo Horizonte - MG - Brasil. \\ ${ }^{3}$ Programa de Pós-Graduação Lato Sensu em Fisiologia e Cinesiologia da Atividade Física e Saúde, \\ da Universidade Gama Filho - Belo Horizonte - MG - Brasil.
}

\begin{abstract}
Resumo: Alguns estudos apontam que indivíduos com unidades músculo-tendíneas com menor amplitude de movimento são capazes de utilizar melhor a energia elástica armazenada na estrutura do tendão em movimentos que envolvem as ações excêntrica e concêntrica. Assim, o objetivo desse estudo foi verificar a influência da flexibilidade de membros inferiores na diferença de desempenho entre o salto Squat Jump (SJ) e o salto Countermovement Jump (CMJ). Doze voluntários do gênero masculino foram submetidos à goniometria de membros inferiores, para avaliação da flexibilidade, na qual foram mensuradas a amplitude de movimento em flexão e extensão de quadril e em dorsiflexão. Posteriormente, foram realizados os testes de saltos verticais e determinada a diferença de desempenho entre os resultados do SJ e os resultados do CMJ. Foi encontrada uma alta correlação inversa ( $r$ $=-0,81, p<0,05)$ entre a amplitude de movimento na flexão de quadril e a melhora do desempenho do SJ para o CMJ. Para a extensão de quadril e dorsiflexão foi encontrada baixa correlação inversa ( $r=-0,45$ e $r=-0,26$ respectivamente, $p<0,05)$. Diante destes resultados foi possível concluir que há um maior aumento do desempenho entre os saltos SJ e CMJ em indivíduos com menor ADM na flexão de quadril. Estes resultados sugerem que indivíduos com menor flexibilidade nos músculos extensores do quadril possuem um maior aproveitamento da energia elástica armazenada durante a ação excêntrica no CMJ.
\end{abstract}

Palavras-Chave: salto contra movimento, flexibilidade, saltos verticais.

Abstract: The elastic properties of muscle, tendon and ligaments play an important role in human movement. The basis of this role is the capacity to store mechanical energy and to return it in elastic recoil. It is discussed that muscular system with less flexibility is able to use stored elastic energy better than muscular system with more flexibility. The aim of this study was to verify the influence of inferior limbs flexibility on Squat Jump (SJ) and Countermovement Jump (CMJ) performance difference. Twelve male volunteers were submitted to inferior limbs goniometry to measure flexibility of hip flexors, hip extensors and of ankle flexors. After that, squat and countermovement jump were realized and the difference between them was calculated. It was found a high and inverse correlation between $(r=-0,81, p<0,05)$ hip extensors flexibility and the improvement in performance from SJ to CMJ. For hip flexors and ankle flexors it was found a low correlation $(r=-0,45$ e $r=-0,26, p<0,05)$. The results suggest that there is a higher increase of performance from SJ to CMJ in subjects with less hip extensors flexibility. It seems that individuals with less hip extensors flexibility can take advantage and use better elastic energy stored in eccentric muscular actions on CMJ.

Key words: countermovement jump, flexibility, vertical jump.

Aceito em 27/11/2008 - Rev. Educ. Fís. 2009 Mar: 144: 30-35. Rio de Janeiro - RJ - Brasil

\section{INTRODUÇÃO}

A flexibilidade pode ser descrita como a amplitude de movimento (ADM) angular de uma articulação ou grupo de articulações (1). Pode ainda ser entendida como um fenômeno complexo, influenciado por componentes biomecânicos (2), neurofisiológicos e anátomo-articulares ${ }^{(3)}$.
O desempenho em alguns esportes pode ser determinado pela capacidade de saltar que o indivíduo possui. As técnicas padronizadas de saltos Squat Jump (SJ) e Countermovement Jump (CMJ) podem ser utilizadas para a análise da capacidade de saltar. O SJ consiste na realização de um salto vertical com meio agachamento, que parte de uma posição estática, sem movimento 
prévio de qualquer segmento, utilizando apenas a ação muscular concêntrica (4). O CMJ consiste na realização do salto vertical a partir da posição ortostática, com contra-movimento prévio, utilizando assim as ações musculares excêntrica e concêntrica.

Segundo Kubo, Kawakami e Fukunaga (5), a seqüência de uma ação excêntrica do músculo, seguida imediatamente por uma ação concêntrica gera um melhor desempenho no salto, devido à energia elástica armazenada. A fase excêntrica de estiramento do conjunto músculo-tendão possibilita um acúmulo de energia elástica, que poderá ser aproveitada, a fim de otimizar a consecutiva ação concêntrica (6). De acordo com Velez ${ }^{(7)}$, a comparação entre o CMJ e o SJ permite a obtenção de um índice que corresponde à diferença percentual na altura obtida entre as duas técnicas de salto. Esta diferença se deve principalmente à energia elástica potencial armazenada nos elementos músculos-tendíneos durante a fase de alongamento. Kreighbaum e Barthels ${ }^{(8)}$ verificaram que a capacidade de gerar força pode aumentar em até $20 \%$ com a participação do ciclo de alongamento-encurtamento.

Bojsen-Moller et al (9) mensuraram a rigidez passiva do complexo tendão-aponeurose do músculo vasto lateral por meio de ultrassonografia. Estes autores identificaram que indivíduos com menor ADM apresentaram melhor desempenho nos saltos SJ e CMJ. Já Kubo et al., (5), não encontraram correlação significativa entre o desempenho em ambas técnicas de salto e a ADM. Entretanto, Kubo et al., ${ }^{(5)}$ utilizaram os a mesma técnica de análise e identificaram que unidades tendíneas com maior rigidez são capazes de utilizar melhor a energia elástica armazenada, em movimentos que envolvem as ações excêntrica e concêntrica. Entretanto, Bazett-Jones, Gibson e McBride (10) não encontram diferenças significativas no desempenho no salto vertical após seis semanas de treinamento de flexibilidade de isquio-surais.

Além do armazenamento de energia elástica, o reflexo do estiramento é outro fator que pode contribuir para otimização da ação concêntrica. Nos músculos existem proprioceptores denominados fusos musculares. Estes proprioceptores detectam a amplitude das alterações no comprimento das fibras musculares, assim como a velocidade de mudança no comprimento dessas fibras (11,12,13). De acordo com Kilani, Palmer, Adrian e Gapsis ${ }^{(14)}$, em alguns casos a resposta mioelétrica pode ser responsável por $85 \%$ do aumento da altura de salto após um contra-movimento.

Diante disso, objetivo desse estudo foi verificar a influência da flexibilidade de membros inferiores na diferença de desempenho do salto SJ para o CMJ.

\section{METODOLOGIA}

\section{Amostra}

A amostra foi composta por 12 voluntários não atletas, homens, com idade média de 28,5 + 5,8 , massa de $74,5+10,9 \mathrm{~kg}$ e estatura $173,5+$ $9,7 \mathrm{~cm}$.

\section{Cuidados éticos}

O projeto deste estudo foi submetido à apreciação do Comitê de Ética da Faculdade Estácio de Sá. Os voluntários foram informados pelos pesquisadores quanto aos objetivos e aos procedimentos metodológicos do estudo, antes da assinatura do Termo de Consentimento Livre e Esclarecido.

\section{Instrumentos}

Foram utilizados neste estudo uma placa de contato e software (Multisprint, versão 3.5.7, Brasil); flexímetro pendular gravitacional (Sanny, precisão de 1 grau, Brasil), balança (R110, Welmy, Brasil) e uma maca. 


\section{Procedimentos}

Todos os voluntários passaram pelo processo de pesagem e mensuração da estatura. Posteriormente foram submetidos à avaliação de flexibilidade de quadril $(F Q)$, extensão de quadril (EQ) e dorsiflexão (DF). Foi utilizada para análise a média dos resultados dos dois membros em cada teste.

Após um intervalo de 30 minutos, os voluntários foram submetidos aos testes de saltos verticais (SJ e CMJ). Cada voluntário executou três saltos SJ e três CMJ para familiarização com a técnica. Em seguida foram realizados os testes, onde cada voluntário realizou três saltos de cada técnica (SJ e CMJ) com intervalo de dois minutos entre cada tentativa. Para análise dos relutados foi considerado o salto de melhor desempenho em cada técnica.

Teste de Flexibilidade: O teste de flexibilidade foi realizado em superfície plana (maca), com o avaliado descalço, sem meias e utilizando roupas que não limitavam seus movimentos.

Para avaliação da ADM em flexão do quadril, o avaliado foi posicionado em decúbito dorsal, fixando-se o joelho do membro que não está sendo avaliado. O segmento não perdia o contato com a maca durante o movimento. O flexímetro foi colocado na face lateral da coxa, para que não houvesse alteração no ângulo com alguma movimentação do joelho. O leitor do flexímetro foi posicionado para o avaliador. Estabilizou-se a pelve, a fim de evitar a elevação do quadril e a retirada da coluna lombar da superfície. Foi realizada a flexão passiva do quadril.

$\mathrm{Na}$ extensão de quadril o avaliado foi posicionado em decúbito ventral com a cabeça voltada lateralmente. O flexímetro foi fixado da mesma forma que na flexão. Estendeu-se o joelho para que a tensão da musculatura anterior não restringisse o movimento. Estabilizou-se a pelve, evitando a rotação ou o balanceio anterior. A crista ilíaca permaneceu em contato com a maca durante a realização do movimento. Foi realizada a extensão passiva do quadril.

$\mathrm{Na}$ avaliação da dorsiflexão o avaliado ficou sentado e os membros em suspensão. O flexímetro foi colocado na face lateral do pé, com o leitor voltado para o avaliador. $\mathrm{O}$ avaliador estabilizou a perna e o pé do avaliado, evitando o movimento do joelho e oferecendo apoio para que o pé não ficasse solto e alterasse a angulação natural.

Saltos Verticais: Durante o SJ o voluntário realizou um salto vertical com meio agachamento que partia de uma posição estática com flexão do joelho de $90^{\circ}$, sem contramovimento prévio de qualquer segmento. As mãos ficaram fixas próximas ao quadril, na região supra-ilíaca; enquanto o tronco ficou na vertical, sem um adiantamento excessivo. Permaneceu estático por dois segundos antes de saltar. Os joelhos permaneceram em extensão durante a fase de vôo.

Para avaliação do CMJ cada voluntário realizou um salto vertical a partir da posição ortostática, mantendo os joelhos em extensão a $180^{\circ}$, com as mãos fixas próximas ao quadril, na região supra-ilíaca. O salto foi realizado com a técnica de contra-movimento, em uma situação específica na qual o atleta executava uma ação excêntrica seguida por uma concêntrica. A flexão do joelho acontecia até o ângulo de $90^{\circ}$, em seguida, o executor fazia a extensão do joelho. O Tronco foi mantido ereto e na vertical sem um adiantamento excessivo. Os joelhos permaneceram em extensão durante a fase de vôo. 
TABELA 1

VALORES DA ADM ENCONTRADOS NA AVALIAÇÃO DA FLEXIBILIDADE E ALTURA DOS SALTOS VERTICAIS

\begin{tabular}{cccccc}
\hline Voluntários & $\begin{array}{c}\text { FQ } \\
\text { (graus) }\end{array}$ & $\begin{array}{c}\text { EQ } \\
\text { (graus) }\end{array}$ & $\begin{array}{c}\text { DF } \\
\text { (graus) }\end{array}$ & $\begin{array}{c}\text { SJ } \\
\text { (cm) }\end{array}$ & $\begin{array}{c}\text { CMJ } \\
\text { (cm) }\end{array}$ \\
\hline $\mathbf{1}$ & 92 & 30 & 27 & 30,7 & 38,9 \\
$\mathbf{2}$ & 107 & 39 & 30 & 33,2 & 37,2 \\
$\mathbf{3}$ & 111 & 47 & 24 & 32,1 & 34,0 \\
$\mathbf{4}$ & 116 & 55 & 27 & 34,7 & 36,7 \\
$\mathbf{5}$ & 106 & 25 & 26 & 33,2 & 36,5 \\
$\mathbf{6}$ & 117 & 56 & 35 & 28,8 & 31,6 \\
$\mathbf{7}$ & 109 & 37 & 24 & 35,6 & 41,5 \\
$\mathbf{8}$ & 105 & 24 & 24 & 26,5 & 27,9 \\
$\mathbf{9}$ & 100 & 49 & 32 & 34,0 & 40,5 \\
$\mathbf{1 0}$ & 134 & 54 & 33 & 28,7 & 29,8 \\
$\mathbf{1 1}$ & 127 & 57 & 38 & 30,2 & 30,3 \\
$\mathbf{1 2}$ & 102 & 25 & 32 & 27,9 & 32,0 \\
\hline Média & $\mathbf{1 1 0}$ & $\mathbf{4 1}$ & $\mathbf{2 9}$ & $\mathbf{3 1 , 3}$ & $\mathbf{3 4 , 7}$ \\
DP & $\mathbf{1 1 , 7}$ & $\mathbf{1 3 , 0}$ & $\mathbf{4 , 9}$ & $\mathbf{2 , 9}$ & $\mathbf{4 , 5}$ \\
\hline
\end{tabular}

FQ - flexão de quadril; EQ - Extensão de quadril; DF - dosiflexão, SJ - squat jump; CMJ - countermovement jump; DP - desvio padrão.

\section{Análise Estatística}

Foi realizada estatística descritiva e inferencial dos dados. Nesta análise foi calculado o percentual de melhora (PM) na altura de salto de cada voluntário no CMJ quando comparado com SJ.

Foi utilizada a correlação de Pearson para correlação dos resultados do teste de flexibilidade e da PM $(p<0,05)$.

\section{RESULTADOS}

Na TABELA 1 estão apresentados os resultados dos testes de flexibilidade e saltos verticais.

Na TABELA2 estão apresentados os percentuais de melhora obtidos por cada voluntário, comparando o desempenho no CMJ com o desempenho obtido no SJ.
TABELA 2

PERCENTUAL DE MELHORA ENTRE OS SALTOS SQUAT JUMP E COUNTERMOVEMENT JUMP

\begin{tabular}{cc}
\hline Voluntários & Melhora do SJ para o CMJ (\%) \\
\hline $\mathbf{1}$ & 21,1 \\
$\mathbf{2}$ & 10,8 \\
$\mathbf{3}$ & 5,6 \\
$\mathbf{4}$ & 5,4 \\
$\mathbf{5}$ & 9,0 \\
$\mathbf{6}$ & 8,9 \\
$\mathbf{7}$ & 14,2 \\
$\mathbf{8}$ & 5,0 \\
$\mathbf{9}$ & 16,0 \\
$\mathbf{1 0}$ & 3,7 \\
$\mathbf{1 1}$ & 0,3 \\
$\mathbf{1 2}$ & 12,8 \\
\hline Média & $\mathbf{9 , 4}$ \\
D.P. & $\mathbf{5 , 9}$ \\
\hline
\end{tabular}

SJ - squat jump; CMJ - countermovement jump; DP - desvio padrão. 
Foi encontrada alta correlação inversa $(r=-0,81)$ entre a ADM em flexão de quadril e o percentual de melhora do SJ para o CMJ. Entre os resultados dos testes de extensão de quadril e o percentual de melhora no desempenho entre os saltos foi encontrada uma baixa correlação inversa $(r=-0,45)$. Também foi encontrada uma baixa correlação inversa $(r=$ $-0,26$ ) entre os resultados dos testes de dorsiflexão e o Percentual de Melhora no desempenho entre os saltos

\section{DISCUSSÃO}

Geralmente o desempenho obtido no CMJ é superior ao desempenho no SJ. Vários fatores contribuem para este aumento no desempenho, mas o principal deles é a utilização de energia elástica armazenada durante a ação excêntrica do CMJ. A utilização desta energia faz com que o indivíduo salte mais alto. Durante o SJ utiliza-se apenas a ação muscular concêntrica, que não propicia a utilização de mecanismos reflexos e elásticos ${ }^{(4,5,6)}$. De acordo com Kreighbaum ${ }^{(8)}$ a capacidade de gerar força pode aumentar em até $20 \%$ com a participação do ciclo de alongamento-encurtamento. Velez ${ }^{(7)}$ aponta que a comparação entre o CMJ e o SJ permite a obtenção de um índice que corresponde à diferença percentual na altura obtida entre as duas técnicas de salto. Esta diferença foi encontra em todos os voluntários deste estudo $(9,4 \pm 5,9 \%)$ e foi aqui denominada como percentual de melhora.

Durante o CMJ o indivíduo deve realizar uma ação excêntrica até $90^{\circ}$ de flexão de joelhos. Levando em consideração o fato de que a flexibilidade pode ser descrita como a amplitude de movimento $\operatorname{articular}^{(1)}$, é possível que indivíduos menos flexíveis em membros inferiores armazenem mais energia elástica quando atingem esta amplitude de $90^{\circ}$. Caso isto ocorra é provável que estes indivíduos apresentem uma maior diferença entre a altura atingida no CMJ e SJ. Desta maneira, deveria existir uma correlação inversa entre a amplitude máxima obtida nos testes de flexibilidade e o percentual de melhora do SJ para o CMJ. Isto ocorreu apenas na flexão de quadril onde foi encontrada alta correlação inversa entre a flexão de quadril e o percentual de melhora do SJ para o CMJ. Assim, os indivíduos que possuem menor flexibilidade nos músculos extensores do quadril apresentaram um percentual de melhora mais elevado. Segundo Taylor ${ }^{(2)}$, devido às características viscoelásticas, quanto maior o estiramento das unidades músculo-tendíneas maior a quantidade de energia elástica armazenada. Assim, músculos com menor flexibilidade, quando estirados com a mesma amplitude tendem a armazenar mais energia elástica.

Estes resultados reforçam os achados de Kubo et al. ${ }^{(5)}$ que identificaram em estruturas músculo-tendíneas com maior rigidez um maior armazenamento e aproveitamento de energia elástica em movimento que envolvem ações excêntricas e concêntricas.

Apesar do SJ também exigir uma flexão de joelhos de $90^{\circ}{ }^{(4)}$, esta é a posição inicial. Como aproveitamento da energia elástica depende da seqüência de uma ação excêntrica do músculo seguida imediatamente por uma ação concêntrica (5), a energia elástica armazenada durante o posicionamento para o SJ é dissipada e a altura atingida neste salto é menor que no CMJ.

De acordo com Kilani et al., (14), a resposta mioelétrica pode ser responsável por $85 \%$ do aumento da altura de salto após um contra-movimento. Quando o músculo é alongado os fusos musculares detectam tanto a velocidade de estiramento quanto a amplitude deste alongamento ${ }^{(11,12,13)}$. Assim, é possível que para atingir a amplitude de $90^{\circ}$ de flexão de joelho no CMJ os músculos mais encurtados sejam alongados com uma maior amplitude. Este fenômeno, além de propiciar um maior armazenamento de energia elástica, pode também desencadear um maior reflexo do estiramento, ou seja, uma maior resposta mioelétrica. 


\section{CONCLUSÃO}

Os resultados desse estudo demonstraram uma alta correlação inversa entre a amplitude de movimento na flexão de quadril e a melhora do desempenho do SJ para o CMJ. Para a extensão de quadril e dorsiflexão foi encontrada baixa correlação inversa. Diante destes resultados foi possível concluir que há um maior aumento no desempenho do salto SJ para o CMJ em indivíduos com menor amplitude de movimento na flexão de quadril. Estes resultados sugerem que indivíduos com menor amplitude de movimento na extensão de quadril provavelmente possuem uma menor flexibilidade nos músculos extensores do quadril e, por isso, possuem maior aproveitamento da energia elástica armazenada durante a ação excêntrica no CMJ.

\section{REFERÊNCIAS BIBLIOGRÁFICAS}

1. Bagrichevsky M. O desenvolvimento da Flexibilidade: Uma análise teórica de mecanismos neurais intervenientes. Revista Brasileira de Ciências do Esporte 2002; 24:199-210.

2. Taylor DC. et al. Viscoelastic properties of muscle-tendon units: The biomechanical effects of stretching. American Journal of Sports Medicine. 1990; 18:300-309.

3. Halbertsma JPK, Ludwig M, Göeken NH. Stretching exercises: effect on passive extensibility and stiffness in short hamstrings of healthy subjects. Arch. Phys. Med. Rehabil 1994; 75:976-981.

4. Bosco, C. et al. New tests for measurement of anaerobic capacity in jumping and leg extensor muscle elasticity. Volleyball, I.F.V.B. Official Magazine 1981;1:22-30.

5. Kubo K, Kawakami Y, Fukunaga T. Influence of elastic properties of tendon structures on jump performance in human. Journal of Applied Physiology 1999: 87;20902096.

6. Bobbert MF, Gerritsen KGM, Litjens MCA, Van Soest AJ. Why is countermovement jump height greater than squat jump height? Medicine and Science in Sports and Exercise 1996:28;1402-1412.

7. Velez M. El entrenamiento de fuerza para mejora del salto. Apuntes Medicina de L'Eport 1992:112;139-156.
8. Kreighbaum E, Barthels KM. Neuromuscular aspects of movement. Biomechanics 1990; 2:63-92.

9. Bojsen-Møller J, Magnusson SP, Rasmussen LR, Kjaer M, Aagaard P. Muscle performance during maximal isometric and dynamic contractions is influenced by the stiffness of the tendinous structures. Journal of Applied Physiology 2005:99;986-994.

10. Bazett-Jones DM, Gibson MH, Mcbride JM. Sprint and vertical jump performances are not affected by six weeks of statics hamstring stretching. Journal of Strength \& Conditioning Research 2008:22;25-32.

11. Bosco C, Viitasalo JT, Komi PV, Luhtanen P. Combined effect of elastic energy and myoelectrical potentiation during stretch-shortening exercise. Acta Physiology 1982: 114;557-565.

12. Stein RB, Kearney RE. Nonlinear behavior of muscles reflexes at the human ankle joint. Journal Neurophysiology 1995:73;65-72.

13. Chen HH, Hippenmeyer S, Arber S, Frank E. Development of the monosynaptic stretch reflex circuit. Current Opinion in Neurobiology 2003: 13;96-102.

14. Kilani HA, Palmer SS, Adrian MJ, Gapsis JJ. Block of the stretch reflex of vastus lateralis during vertical jumps. Human Movement Science 1989: 8;247-269.

\section{Endereço:}

Rua Genoveva de Souza, 1241, apto. 502.

Bairro Sagrada Família, Belo Horizonte, Minas Gerais, Brasil.

CEP: $31030-220$

Telefone: (0xx31) 3461-6928

E-mail: brunopena@yahoo.com.br 